\title{
DISKUSSION
}

\section{Konsens- oder mehrheitsdemokratischer Wandel des Parlamentarismus? Eine Replik auf Gerd Strohmeier in Heft 3/2007 der ZParl*}

\author{
Frank Decker
}

Vorschläge zur Reform politischer Institutionen sind nur dann tauglich und genügen politikwissenschaftlichen Kriterien, wenn man ihre Systemverträglichkeit beachtet. Daraus lassen sich drei Mindestanforderungen an eine seriöse Analyse ableiten. Erstens gilt es zu fragen, ob die Institutionen zu den gesellschaftlichen und kulturellen Grundlagen des Regierungssystems passen beziehungsweise mit diesen vereinbar sind. Zweitens muss untersucht werden, ob und auf welche Weise sich die Änderung einer institutionellen Systemeigenschaft auf andere Systemeigenschaften auswirkt. Beides lässt sich am besten mittels einer länder- oder systemvergleichenden Betrachtung herausfinden. Drittens geht es um die Umsetzbarkeit der Vorschläge. Hier müssen sowohl die historischen Prägungen berücksichtigt werden, die den Pfad möglicher Veränderungen vorgeben, als auch die Interessenlagen der politischen Akteure. Es reicht nicht, sich auf der grünen Wiese Idealmodelle auszumalen und dann an die Politik zu appellieren, sie möge diese doch bitte verwirklichen. Analysen, die so argumentieren, bleiben ganz im Voluntaristischen befangen und können allenfalls den heuristischen Wert einer Utopie für sich reklamieren.

Das Plädoyer, das Gerd Strohmeier im letzten Heft der ZParl für die Einführung einer gemäßigten Variante der Mehrheitswahl im deutschen Regierungssystem abgegeben hat, wird keiner der genannten Anforderungen gerecht. Strohmeier folgt dem Lehrbuchwissen der Politologie darin, dass ein Wahlsystem grundsätzlich zwei Funktionen hat. Zum einen soll es die Bildung einer stabilen Mehrheitsregierung ermöglichen, zum anderen eine möglichst gerechte Repräsentation der Wählerpräferenzen gewährleisten. Während die erstgenannte Bedingung nach allgemeiner Meinung von einem Mehrheitswahlsystem wesentlich besser erfüllt wird als von einem Verhältniswahlsystem, widerspricht Strohmeier der verbreiteten Ansicht, dass ein Verhältniswahlsystem die Wählerpräferenzen genauer und mithin gerechter widerspiegele als ein Mehrheitswahlsystem; dies gelte allenfalls auf der parlamentarischen Ebene, nicht aber bei der Regierungsbildung, wo die eigentlichen Wahlsieger unter Umständen zu Verlierern mutieren und kleine Parteien einen ihnen nicht gebührenden Einfluss ausüben könnten (S. 584 f.). Daraus ergibt sich in der Summe eine klare normative Präferenz des Autors für die Mehrheitswahl.

Möchte man der Analyse bis zu diesem Punkt noch folgen, so muss Strohmeier entschieden widersprochen werden, wenn er die Fähigkeit zur Regierungsbildung ohne weiteres mit

Gerd Strohmeier, Ein Plädoyer für die „gemäßigte Mehrheitswahl“: optimale Lösung für Deutschland, Vorbild für Österreich und andere Demokratien, in: ZParl, 38. Jg. (2007), H. 3, S. 578 ff. 
Regierungsfähigkeit oder Regierbarkeit gleichsetzt. Gewiss hat eine Regierung mit breiter parlamentarischer Mehrheit nur einer Partei bessere Möglichkeiten als eine Koalitionsregierung, ihr Programm durchzusetzen und damit auch unpopuläre Reformen herbeizuführen. Dies gilt zumal, wenn im Gesetzgebungsprozess keine weiteren Vetospieler vorhanden sind, die ihre Vorhaben gegebenenfalls durchkreuzen könnten. Daraus aber den Schluss zu ziehen, das mehrheitsdemokratische System sei seinen konsensdemokratischen Gegenstücken in punkto Regierungsfähigkeit generell überlegen, ist verkehrt. Die Befunde der empirischen Politikforschung deuten in mancherlei Hinsicht eher in die gegenteilige Richtung, auch wenn das Lob des konsensdemokratischen Modells unter dem Einfluss der LijphartSchule in der Komparatistik gelegentlich übertrieben worden ist. ${ }^{1}$ So werden Hoffnungen in die Mehrheitswahl gesetzt, die diese bei nüchterner Betrachtung nicht rechtfertigen kann.

Wie wenig die Annahmen des Autors stimmen, kann man an seiner Analyse des deutschen Falles ablesen. Hier wird die seit 2005 amtierende Große Koalition apodiktisch als Ursache von Politikblockaden und Reformstillstand gewertet (S. 582). Trotz des zutreffenden, an mehreren Bespielen festgemachten Hinweises auf die Schwierigkeiten der regierungsinternen Kompromissfindung handelt es sich dabei jedoch noch nicht einmal um die halbe Wahrheit. Wenn es in der Vergangenheit eine Ursache für Politikblockaden gegeben hat, so waren es die gegenläufigen Mehrheitsverhältnisse zwischen Bundestag und Bundesrat, die den Oppositionsparteien die Möglichkeit einräumten, Regierungsvorhaben zu behindern oder im eigenen Sinne zu verändern. Gerade dieses Problem ist aber durch das Zusammengehen der beiden großen Parteien entschärft worden. Maßnahmen wie die Kürzung von Steuersubventionen oder die Erhöhung der Mehrwertsteuer, die maßgeblich zur Konsolidierung des Bundeshaushaltes beigetragen haben, wären ansonsten nicht durchsetzbar gewesen. Die Große Koalition dürfte daher in der Summe zu einer Verbesserung der Regierungsfähigkeit geführt haben.

Dass Strohmeier die Existenz des Vetospielers Bundesrat in seinem Plädoyer für das Mehrheitswahlsystem vollständig ausblendet, ist kein Zufall. Dessen Einführung auf Bundesebene würde nämlich am Problem der doppelten Mehrheiten nichts ändern, im Gegenteil: Wenn die kleinen Parteien im Bund so stark an den Rand gedrängt werden, dass sie für die Regierungsbildung keine Rolle mehr spielen, wie es Strohmeiers Vorschlag eines Mehrheitswahlsystems mit proportionaler Zusatzliste intendiert, dann wäre es auch nicht sinnvoll, sie auf der gliedstaatlichen Ebene weiter an den Regierungen zu beteiligen. In den Ländern entstünde deshalb ein Druck, die eigenen Wahlrechtsregelungen jenen des Bundes anzupassen, wie es bereits in der Vergangenheit regelmäßig geschehen ist. Aufgrund des bei Landtagswahlen auftretenden Zwischenwahleffekts hätte das mit ziemlicher Sicherheit abweichende Mehrheitsverhältnisse zur Folge. Dies gilt auch nach der 2006 in Kraft getretenen Föderalismusreform, die den Anteil der zustimmungsbedürftigen Gesetze nicht - jedenfalls noch nicht - in dem erhofften Ausmaß gesenkt hat. ${ }^{2}$ Bei „einfarbigen“ Mehrheiten

1 Dazu die Kritik von André Kaiser, Alternanz und Inklusion. Zur Repräsentation politischer Präferenzen in den westeuropäischen Demokratien, 1950 - 2000, in: ders. / Thomas Zittel (Hrsg.), Demokratietheorie und Demokratieentwicklung. Festschrift für Peter Graf Kielmansegg, Wiesbaden 2004, S. $173 \mathrm{ff}$.

2 Vgl. dazu den Beitrag von Marcus Höreth in diesem Heft der ZParl. 
wäre die Kompromissbildung aber noch schwieriger als in der Vergangenheit. Die Interessengegensätze zwischen Regierung und Opposition beziehungsweise Bund und Ländern, die durch das jetzige System vielfältiger und disparater Koalitionen zumindest abgemildert werden können, würden unvermittelt aufeinanderprallen.

Ein Mehrheitswahlrecht wäre nicht nur institutionell mit den Strukturen des deutschen Parteienbundesstaates unvereinbar, es würde sich auch von den gesellschaftlichen Grundlagen des Parteiensystems unzulässig entfernen. Seine Einführung soll ja gerade der zunehmenden Fragmentierung des Parteiensystems einen Riegel vorschieben, die wir in der Bundesrepublik seit zwei Jahrzehnten feststellen. Konnten die beiden großen Parteien bis zu Beginn der 1980er Jahre zusammengenommen noch über 90 Prozent der Wählerstimmen auf sich vereinigen, so fiel dieser Anteil bei der jüngsten Bundestagswahl auf unter siebzig. Gleichzeitig stieg der zusammengefasste Stimmenanteil der kleinen Parteien auf 30 Prozent an, von denen heute gleich drei im Parlament vertreten sind. Bis zur Bundestagswahl 2005 bereitete die Pluralisierung des Parteiensystems keine größeren Probleme, da die Wahlergebnisse die Bildung einer kleinen (Zweier-)Koalition nach dem vertrauten Muster stets ermöglichten. Erst mit der Herausbildung einer Fünf-Parteien-Struktur im Zuge der erfolgreichen Westausdehnung der PDS verdichtete sich zur Gewissheit, dass die Ära des mehrheitsdemokratischen Parlamentarismus auf absehbare Zeit vergangen ist. Obwohl man diese Entwicklung hätte vorausahnen können, traf sie die politischen Akteure unvorbereitet. Ihre Konsequenz war die Bildung einer Großen Koalition, in die sich die beiden Volksparteien jedoch nur widerstrebend fügten, nachdem sie das gegnerschaftliche Prinzip des Parteienwettbewerbs jahrzehntelang internalisiert hatten. Wie schwer ihnen die Abkehr vom mehrheitsdemokatischen Modell fiel, zeigte sich daran, dass am Wahlabend auch über andere Regierungsformate offen spekuliert wurde. Diese waren zuvor noch mit einem Tabu belegt worden, weil sie der Stabilitätsorientierung des parlamentarischen Systems scheinbar widersprachen.

Die Einführung eines Mehrheitswahlsystems in der von Strohmeier vorgeschlagenen Form, die auf die Wiederherstellung der Regierungsstabilität zielt, würde bedeuten, dass der heutige Mandatsanteil der Oppositionsparteien im Bundestag von etwa 30 Prozent mit einem Schlag auf fünf bis maximal zehn Prozent reduziert würde. Ein so radikaler Schnitt wäre mit Blick auf die tatsächliche Entwicklung der parteipolitischen Kräfteverhältnisse nicht legitimierbar. Er würde das bewirken, was der Autor selbst als unerwünschten Nebeneffekt des Verhältniswahlrechts kritisiert: nämlich die Verlierer (die großen Parteien) zu Gewinnern machen. Dass ein solches Vorhaben der Öffentlichkeit schwer begründet werden könnte, liegt auf der Hand. Es würde nicht nur den erbitterten Widerstand der Oppositionsparteien Bündnis 90/Grüne, FDP und Linke hervorrufen, die durch den Übergang zum Mehrheitswahlsystem zur Bedeutungslosigkeit verurteilt wären, sondern auch der verfassungspolitischen Gewohnheit in diesem Lande widersprechen, Wahlrechtsreformen, die ja im Unterschied zu Verfassungsänderungen keiner qualifizierten Mehrheit bedürfen, tunlichst im Konsens aller betroffenen Parteien vorzunehmen. ${ }^{3}$ Hierin hat sich die Bundesrepublik bisher immer vorteilhaft von der manipulativen Praxis anderer Länder unterschieden (etwa Italien oder Frankreich). Deshalb reicht es nicht aus, die Einführbarkeit eines Mehr-

3 Vgl. Frank Decker, Parlamentarische Demokratie versus Volksgesetzgebung. Der Streit um ein neues Wahlrecht in Hamburg, in: ZParl, 38. Jg. (2007), H. 1, S. 129 ff. 
heitswahlsystems allein an die strukturelle Chancengleichheit der beiden großen Parteien zu knüpfen (S. 590). ${ }^{4}$

Völlig an der Sache vorbei geht in diesem Zusammenhang die vom Autor bemühte Parallele zur Wahlrechtsdiskussion in Großbritannien (S. 587). Überlegungen, das britische Wahlrecht in Richtung einer größeren Proportionalität zu verändern, mögen sich zwar mit dem von Strohmeier für die Bundesrepublik propagierten Modell treffen, verfolgen aber gerade das entgegengesetzte Ziel, nämlich das Wahlsystem an die veränderten gesellschaftlichen Gegebenheiten anzupassen. Nachdem die Bindungskraft der beiden großen Parteien rapide gesunken ist, schwindet die Legitimationsbasis des überkommenen Mehrheitswahlsystems in Großbritannien zunehmend. Auch in anderer Hinsicht ist das Westminster-Modell im Rückzug begriffen. Insofern haben wir es heute, was die institutionelle Entwicklung der Regierungssysteme in Europa betrifft, mit einer bemerkenswerten Ungleichzeitigkeit zu tun. Während konsensdemokratische Länder wie Österreich, die Niederlande oder die Schweiz ihre Konkordanzstrukturen in der Vergangenheit zugunsten einer stärkeren Wettbewerbsorientierung der Parteien zurückgedrängt haben, wird die britische Politik gewaltenteiliger und konsensueller. Vor diesem Hintergrund mutet es anachronistisch an, wenn Autoren wie Strohmeier sich bei der Reform des eigenen Regierungssystems an einem Lehrbuchmodell orientieren, das auch im Mutterland des mehrheitsdemokratischen Parlamentarismus längst nicht mehr besteht. ${ }^{5}$

Dies bedeutet selbstverständlich nicht, dass im deutschen Regierungssystem alles zum Besten bestellt wäre und sämtliche Reformversuche von vornherein unterbleiben sollten. Legt man die Kategorien Arend Lijpharts zugrunde, stellt die Bundesrepublik eine Mischform zwischen Mehrheits- und Konsensdemokratie dar. ${ }^{6}$ Theoretisch bestünde damit die Möglichkeit, die institutionellen Strukturen in die eine oder andere Richtung fortzuentwickeln. Bei der Föderalismusreform haben die Verfassunggeber den mehrheitsdemokratischen Weg beschritten, wobei ihnen allerdings kein Erfolg beschieden war. ${ }^{7}$ Die Pluralisierung des Parteiensystems erzwingt wiederum einen stärkeren Konsensualismus auf der parlamentarischen Ebene, der sich in einer Öffnung der politischen Akteure für neue Koalitionsmuster und Regierungsformate niederschlägt. Das Spektrum reicht dabei von verschiedenen Dreier-Bündnissen über gestützte oder geduldete Minderheitsregierungen bis hin zu Großen Koalitionen. ${ }^{8}$

Das demokratische Problem des Konsensmodells liegt darin, dass es den Einfluss des Wählers auf die Regierungsbestellung begrenzt, die hier zumeist ganz den Parteien beziehungsweise Fraktionen und ihren Führungen überlassen bleibt. Im speziellen Fall der Großen Koalition kommt hinzu, dass diese wesentliche Funktionsbedingungen des Parlamentarismus untergräbt, indem sie die Gewaltenbalance einseitig zugunsten der Regie-

4 Nachdem die SPD durch die Etablierung einer gesamtdeutschen Linken gegenüber der Union in eine Minderheitenposition geraten ist, wäre selbst diese Voraussetzung zurzeit nicht gegeben.

5 Vgl. Tony Wright, British Politics. A Very Short Introduction, Oxford 2003, S. 53 ff.

6 Vgl. Everhard Holtmann / Helmut Voelzkow (Hrsg.), Zwischen Wettbewerbs- und Verhandlungsdemokratie. Analysen zum Regierungssystem der Bundesrepublik Deutschland, Wiesbaden 2000.

7 Vgl. Fritz W. Scharpf, Föderalismusreform: Weshalb wurde so wenig erreicht?, in: APuZ, B 50 (2006), S. 6 - 11.

8 Vgl. Frank Decker, Die Bundesrepublik auf der Suche nach neuen Koalitionen, in: APuZ, B 35-36 (2007), S. 26 - 33. 
rung(smehrheit) verschiebt. Die Frage nach demokratischen Korrektiven stellt sich dann in der Tat. Die Antwort kann aber nicht in einer Wahlrechtsreform liegen, sondern - wenn überhaupt - in einer Stärkung der parlamentarischen Kontrollrechte oder der Einführung beziehungsweise dem Ausbau direktdemokratischer Entscheidungsformen zur Ergänzung der bisher ausschließlich parlamentarischen Repräsentation. ${ }^{9}$

Auch beim Wahlrecht braucht in der Bundesrepublik längst nicht alles beim Alten zu bleiben. Wenn die Legitimationskraft des derzeitigen Modells schwindet und Parteien wie Parlamente an zunehmender Repräsentationsschwäche leiden, dann liegt es nahe, die personellen Auswahlkomponenten des Wahlsystems zu Lasten der reinen Parteienwahl zu verstärken, wie es auf der kommunalen Ebene heute weitgehend schon geschieht. Im letzten Heft der ZParl sind dazu in einem anderen Aufsatz interessante Vorschläge gemacht worden. ${ }^{10}$ Diese genügen im Unterschied zu dem von Strohmeier vorgeschlagenen Modell den oben genannten Anforderungen an eine institutionelle Reform, weil sie innerhalb der vorhandenen Grundstruktur eines personalisierten Verhältniswahlsystems mit Sperrklausel verbleiben.

Nachdem die Idee der Mehrheitswahl in der Vergangenheit zumeist nur noch von Altpolitikern hochgehalten wurde (wie dem verstorbenen Rainer Barzel), die damit einer unter der ersten Großen Koalition (1966 bis 1969) aus ihrer Sicht verpassten Chance nachtrauerten, hat sie in den letzten Jahren eine gewisse Renaissance erfahren. Im Bemühen, die Ursachen der bundesdeutschen Reformmalaise zu beschreiben, haben insbesondere Journalisten die angeblich zu konsenslastigen Strukturen des politischen Systems als Übel ausgemacht. ${ }^{11}$ Die Forderung nach „Entkomplizierung“ der Entscheidungsabläufe, die hinter der Apologie des mehrheitsdemokratischen Modells steht, dürfte gewiss vielen Menschen aus der Seele sprechen. Sogar der Präsident des Bundesverfassungsgerichts hat sie sich zu Eigen gemacht. ${ }^{12}$ Dabei handelt es sich aber um eine extrem verkürzte, populistische Sichtweise, der gerade die Politikwissenschaft aufklärerisch entgegenwirken sollte (statt sie durch unpassende Reformvorschläge selbst zu befördern).

9 Vgl. ders., Plebiszitäre Elemente ins Grundgesetz? Anmerkungen zu einer stagnierenden Debatte, in: MUT. Forum für Kultur, Politik und Geschichte, 42. Jg. (2007), Nr. 479, S. 6 - 16.

10 Heiko Franke / Andreas Grimmel, Wahlen mit System? Reformüberlegungen zur personalisierten Verhältniswahl, in: ZParl, 38. Jg. (2007), H. 3, S. 591 - 602.

11 Vgl. zum Beispiel Thomas Darnstädt, Konsens ist Nonsens. Wie die Republik wieder regierbar wird, München 2006.

12 Hans-Jürgen Papier, Überholte Verfassung?, in: Frankfurter Allgemeine Zeitung vom 23. November 2003, S. 8. 